\title{
DISTRIBUTION OF POLYNOMIALS WITH CYCLES OF A GIVEN MULTIPLIER
}

\author{
GIOVANNI BASSANELLI AND FRANÇOIS BERTELOOT
}

\begin{abstract}
In the space of degree $d$ polynomials, the hypersurfaces defined by the existence of a cycle of period $n$ and multiplier $e^{i \theta}$ are known to be contained in the bifurcation locus. We prove that these hypersurfaces equidistribute the bifurcation current. This is a new result, even for the space of quadratic polynomials.
\end{abstract}

\section{$\S 1$. Introduction}

In a holomorphic family $\left(f_{\lambda}\right)_{\lambda \in M}$ of rational maps, the sets $\operatorname{Per}_{n}(w)$ of parameters for which $f_{\lambda}$ has a cycle of exact period $n$ and multiplier $w$ turn out to be hypersurfaces. One knows, since the fundamental work of Mañé, Sad, and Sullivan [15], that the closure of the union of the hypersurfaces $\operatorname{Per}_{n}\left(e^{i \theta}\right)$ coincides with the bifurcation locus $\mathcal{B i f}(M)$, that is, the set of parameters $\lambda_{0}$ for which the dynamics of $f_{\lambda_{0}}$ drastically change under small perturbation. Our aim here is to describe precisely, from a measure-theoretic point of view, the asymptotic behavior of $\operatorname{Per}_{n}\left(e^{i \theta}\right)$ as the period $n$ grows.

Our main tool is the bifurcation current $T_{\text {bif }}$ introduced by DeMarco [8]. It is a positive, closed $(1,1)$-current supported by $\mathcal{B i f}(M)$ which admits both the Lyapunov function $L(\lambda)$ and the sum of values of the Green function on critical points as potentials (see [9] or [1]):

$$
T_{\mathrm{bif}}=d d^{c} L(\lambda)=d d^{c} \sum G_{\lambda}\left(c_{\lambda}\right)
$$

The bifurcation current and its powers $T_{\text {bif }}^{k}\left(k \leq \operatorname{dim}_{\mathbf{C}} M\right)$ have been used in several recent works (see [1], [12], [11], [6], [2]) devoted to the study of measurable or complex analytic properties of the bifurcation locus. In particular, Dujardin and Favre ([12, Theorems 1 and 4.2]) have used the Greenlike potentials to get equidistribution results concerning the hypersurfaces of $M$ defined by the preperiodicity of a critical point.

Received May 13, 2009. Accepted March 25, 2010.

2000 Mathematics Subject Classification. Primary 37F45; Secondary 32U15.

(C) 2011 by The Editorial Board of the Nagoya Mathematical Journal 
In order to study the asymptotic distribution of the hypersurfaces $\operatorname{Per}_{n}(w)$, it is more convenient to use the Lyapunov function since $L(\lambda)$ is well related to the multipliers of $n$-periodic repelling cycles (see Theorem 2.3). This dynamical property allows us to compare $L(\lambda)$ with $d^{-n} \ln \left|p_{n}(\lambda, w)\right|$, where the functions $p_{n}(\cdot, w)$ canonically define the hypersurfaces $\operatorname{Per}_{n}(w)$. Setting $\left[\operatorname{Per}_{n}(w)\right]:=d d^{c} \ln \left|p_{n}(\lambda, w)\right|$ and using basic potential-theoretic tools, we get the following general equidistribution statements.

THEOREM 1.1. Let $T_{\text {bif }}$ be the bifurcation current of some holomorphic family of rational maps $\left(f_{\lambda}\right)_{\lambda \in M}$. Then

$$
\begin{aligned}
& d^{-n}\left[\operatorname{Per}_{n}(w)\right] \rightarrow T_{\text {bif }}, \quad \text { when }|w|<1, \\
& \frac{d^{-n}}{2 \pi} \int_{0}^{2 \pi}\left[\operatorname{Per}_{n}\left(r e^{i \theta}\right)\right] d \theta \rightarrow T_{\text {bif }}, \quad \text { when } r \geq 0, \text { and } \\
& d^{-n} d d_{(\lambda, w)}^{c} \ln \left|p_{n}(\lambda, w)\right| \rightarrow d d^{c} L(\lambda),
\end{aligned}
$$

where the convergence is weak, occurs in $M$ and, for the last statement, in $M \times \mathbf{C}$.

The first two assertions are quite easily obtained and were actually essentially given in [2] (the case $w=0$ is also covered by Dujardin and Favre's result).

When $|w|=1$, the convergence of $d^{-n}[n(w)]$ would easily follow from our arguments if the density of hyperbolic parameters were known. Our basic observation is that the same conclusion occurs when $M$ is a Riemann surface, in which the set of nonhyperbolic parameters is compact and the bifurcation locus is contained in the closure of hyperbolic parameters (see Proposition 3.4). This already covers the case of the family of quadratic polynomials. To go further, our strategy is therefore to slice $M$ with hypersurfaces which are chosen for the good repartition of hyperbolic parameters. The existence of such slices is intimately related to the behavior at infinity of the bifurcation locus, and for this reason we work in the family of degree $d$ polynomials. In this family, the control we need actually follows from the work of Branner and Hubbard [5] on the compactness of the connectedness locus (see Theorem 4.2). Our main result is the following.

THEOREM 1.2. Let $d \geq 2$, and let $\left\{P_{c, a}\right\}_{(c, a) \in \mathbf{C}^{d-1}}$ be the holomorphic family of degree $d$ polynomials parameterized by defining $P_{c, a}$ as the polynomial of degree $d$ whose critical points are $\left(0, c_{1}, \ldots, c_{d-2}\right)$ and such that $P_{c, a}(0)=a^{d}$. 
Let $T_{\text {bif }}$ be the bifurcation current of this family. Then $\lim _{n} d^{-n}\left[\operatorname{Per}_{n}(w)\right]=$ $T_{\text {bif }}$ for any $w$ such that $|w| \leq 1$.

\section{$\S 2$. Some tools}

\subsection{Hypersurfaces $\operatorname{Per}_{n}(w)$}

For any holomorphic family of rational maps, the following result describes precisely the set of maps having a cycle of given period and multiplier.

Theorem 2.1. Let $f: M \times \mathbf{P}^{1} \rightarrow \mathbf{P}^{1}$ be a holomorphic family of degree $d \geq 2$ rational maps. Then for every integer $n \in \mathbf{N}^{*}$, there exists a holomorphic function $p_{n}$ on $M \times \mathbf{C}$ which is polynomial on $\mathbf{C}$ and such that

(1) for any $w \in \mathbf{C} \backslash\{1\}$, the function $p_{n}(\lambda, w)$ vanishes if and only if $f_{\lambda}$ has a cycle of exact period $n$ and multiplier $w$;

(2) $p_{n}(\lambda, 1)=0$ if and only if $f_{\lambda}$ has a cycle of exact period $n$ and multiplier 1 or a cycle of exact period $m$ whose multiplier is a primitive rth root of unity with $r \geq 2$ and $n=m r$;

(3) for every $\lambda \in M$, the degree $N_{d}(n)$ of $p_{n}(\lambda, \cdot)$ satisfies $d^{-n} N_{d}(n) \sim 1 / n$.

This leads to the following definitions. For any integer $n$ and any $w \in \mathbf{C}$, the subset $\operatorname{Per}_{n}(w)$ of $M$ is the hypersurface given by

$$
\operatorname{Per}_{n}(w):=\left\{\lambda \in M / p_{n}(\lambda, w)=0\right\}
$$

and, taking into account the possible multiplicities, we consider the following integration currents:

$$
\left[\operatorname{Per}_{n}(w)\right]:=d d_{\lambda}^{c} \ln \left|p_{n}(\lambda, w)\right| .
$$

Let us briefly recall the construction of the functions $p_{n}$. (For more details, we refer the reader to Milnor [16] or Silverman [17, Chapter 4].)

One first constructs the dynatomic polynomials $\Phi_{\varphi, n}^{*}$ associated to a rational map $\varphi$ of degree $d \geq 2$. Let us denote by $F^{n}=\left(F_{1}^{n}, F_{2}^{n}\right)$ the iterates of some lift $F$ of $\varphi$ to $\mathbf{C}^{2}$, and let us define homogeneous polynomials $\Phi_{\varphi, n}$ on $\mathrm{C}^{2}$ by setting

$$
\Phi_{\varphi, n}(X, Y):=Y F_{1}^{n}(X, Y)-X F_{2}^{n}(X, Y) .
$$

The divisor $\operatorname{Div}\left(\Phi_{\varphi, n}\right)$ induced by $\Phi_{\varphi, n}(X, Y)$ on $\mathbf{P}^{1}$ is precisely the set of periodic points of $\varphi$ with exact period dividing $n$. Denoting $\mu$ the classical Möbius function, one then sets

$$
\Phi_{\varphi, n}^{*}(X, Y):=\prod_{k \mid n}\left(\Phi_{\varphi, n}(X, Y)\right)^{\mu(n / k)} .
$$


Using the fact that the sum $\sum_{k \mid n} \mu(k / n)$ vanishes if $n>1$ and that it is equal to 1 if $n=1$, one may show that $\Phi_{\varphi, n}^{*}$ is a polynomial whose degree $\nu_{d}(n)$ depends only on $n$ and $d$. The divisor $\operatorname{Div}\left(\Phi_{\varphi, n}^{*}\right)$ induced by $\Phi_{\varphi, n}^{*}(X, Y)$ on $\mathbf{P}^{1}$ clearly contains the periodic points of $\varphi$ with exact period equal to $n$. The other points contained in $\operatorname{Div}\left(\Phi_{\varphi, n}^{*}\right)$ are precisely the periodic points of $\varphi$ whose exact period $m$ divides $n(m=n r, r \geq 2)$ and whose multiplier is a primitive $r$ th root of unity (see [17, Theorem 4.5, p. 151]).

If $z \in \operatorname{Div}\left(\Phi_{\varphi, n}^{*}\right)$ has exact period $m$ with $n=m r$, then we will denote by $w_{n}(z)$ the $r$ th power of the multiplier of $z$ (i.e., $\left(\varphi^{n}\right)^{\prime}(z)$ in good coordinates). One sees in particular that the following fact occurs: a point $z$ is periodic of exact period $n$ and $w_{n}(z) \neq 1$ if and only if $z \in \operatorname{Div}\left(\Phi_{\varphi, n}^{*}\right)$ and $w_{n}(z) \neq 1$.

Let us now consider the sets

$$
\Lambda_{n}^{*}(\varphi):=\left\{w_{n}(z) ; z \in \operatorname{Div}\left(\Phi_{\varphi, n}^{*}\right)\right\},
$$

where the points in $\operatorname{Div}\left(\Phi_{\varphi, n}^{*}\right)$ are counted with multiplicity, and let us denote by $\sigma_{i}^{*(n)}(\varphi), 1 \leq i \leq \nu_{d}(n)$ the associated symmetric functions. We define the polynomials $p_{n}(\varphi, w)$ by

$$
\left(p_{n}(\varphi, w)\right)^{n}:=\prod_{i=0}^{\nu_{d}(n)} \sigma_{i}^{*(n)}(\varphi)(-w)^{\nu_{d}(n)-i},
$$

and therefore $p_{n}(\varphi, w)=0$ if and only if $w \in \Lambda_{n}^{*}(\varphi)$. The properties of $p_{n}$ follow easily from this construction. The degree $N_{d}(n)$ of $p_{n}(\lambda, \cdot)$ is equal to $(1 / n) \nu_{d}(n)=(1 / n) \sum_{k \mid n} \mu(n / k) d^{k}$. In particular, $d^{-n} N_{d}(n) \sim 1 / n$.

\subsection{Lyapunov exponent and bifurcation current}

Every rational map of degree $d \geq 2$ on the Riemann sphere admits a maximal entropy measure $\mu_{f}$. The Lyapunov exponent of $f$ with respect to the measure $\mu_{f}$ is given by $L(f)=\int_{\mathbf{P}^{1}} \log \left|f^{\prime}\right| \mu_{f}$ (see [10] for a general exposition in any dimension).

When $f: M \times \mathbf{P}^{1} \rightarrow \mathbf{P}^{1}$ is a holomorphic family of degree $d$ rational maps, the Lyapunov function $L$ on the parameter space $M$ is defined by

$$
L(\lambda)=\int_{\mathbf{P}^{1}} \log \left|f_{\lambda}^{\prime}\right| \mu_{\lambda},
$$

where $\mu_{\lambda}$ is the maximal entropy measure of $f_{\lambda}$. The function $L$ is plurisubharmonic (PSH) on $M$, and the bifurcation current $T_{\text {bif }}$ of the family is a closed, positive $(1,1)$-current on $M$ which may be defined by

$$
T_{\mathrm{bif}}:=d d^{c} L(\lambda)
$$


As it has been shown by DeMarco [9], the support of $T_{\text {bif }}$ coincides with the bifurcation locus of the family in the sense of Mañé, Sad, and Sullivan [15] (see also [1, Theorem 5.2]).

Let us recall that Mañé, Sad, and Sullivan have shown that the complement of the bifurcation locus is a dense open subset of the parameter space $M$ whose connected components are called stable components. They have also shown that any neutral cycle is persistent on the stable components. In the language of Theorem 2.1, this property may be expressed as follows.

REMARK 2.2. For $\left|w_{0}\right|=1$, a function $p_{n}\left(\lambda, w_{0}\right)$ either does not vanish on any stable component or vanishes identically on $M$.

In our study, we combine classical potential-theoretic methods with the following dynamical property (see [3] or [4], where this has been proved for endomorphisms of $\mathbf{P}^{k}$ ).

TheOREM 2.3. Let $f: \mathbf{P}^{1} \rightarrow \mathbf{P}^{1}$ be a degree $d \geq 2$ rational map, let $\mu$ be its maximal entropy measure, and let $L$ be the Lyapunov exponent of $f$ with respect to $\mu$. Then

$$
L=\lim _{n} \frac{d^{-n}}{n} \sum_{p \in R_{n}^{*}} \ln \left|\left(f^{n}\right)^{\prime}(p)\right|,
$$

where $R_{n}^{*}:=\left\{p \in \mathbf{P}^{1} / p\right.$ has exact period $n$ and $\left.\left|\left(f^{n}\right)^{\prime}(p)\right|>1\right\}$.

The continuity of the Lyapunov function will also play a crucial role. This was proved by Mañé [14], but a simple argument based on DeMarco's formula shows that this function is actually Hölder-continuous (see [1, Corollary 3.4$]$ ).

Theorem 2.4. Let $f: M \times \mathbf{P}^{1} \rightarrow \mathbf{P}^{1}$ be a holomorphic family of degree $d \geq 2$ rational maps. Let $L(\lambda)$ be the Lyapunov exponent of $\left(\mathbf{P}^{1}, f_{\lambda}, \mu_{\lambda}\right)$, where $\mu_{\lambda}$ is the maximal entropy measure of $f_{\lambda}$. Then the function $L(\lambda)$ is Hölder-continuous on $M$.

We end this section by recalling a well-known compactness principle for subharmonic functions which will be used frequently in the paper.

THEOREM 2.5. Let $\left(\varphi_{j}\right)$ be a sequence of subharmonic functions which is locally uniformly bounded from above on some domain $\Omega \subset \mathbf{R}^{n}$. If $\left(\varphi_{j}\right)$ does not converge to $-\infty$, then a subsequence $\left(\varphi_{j_{k}}\right)$ converges in $L_{\text {loc }}^{1}(\Omega)$ to some subharmonic function $\varphi$. In particular, $\left(\varphi_{j}\right)$ converges in $L_{\mathrm{loc}}^{1}(\Omega)$ to some subharmonic function $\varphi$ if it converges pointwise to $\varphi$. 


\section{§3. Distribution of $\operatorname{Per}_{n}(w)$ in general families}

In this section, we consider an arbitrary holomorphic family $f: M \times \mathbf{P}^{1} \rightarrow$ $\mathbf{P}^{1}$ of degree $d \geq 2$ rational maps. We investigate the convergence of the currents $\left(1 / d^{n}\right)\left[\operatorname{Per}_{n}(w)\right]$ towards the bifurcation current $T_{\text {bif }}$ by considering the sequences of their potentials, and therefore compare the Lyapunov function $L$ with the limits of $\left(1 / d^{n}\right) \ln \left|p_{n}(\lambda, w)\right|$, where $p_{n}(\lambda, w)$ are the polynomials given by Theorem 2.1 .

This leads us to consider the following sequences of PSH functions

$$
\begin{aligned}
L_{n}^{r}(\lambda) & :=\frac{d^{-n}}{2 \pi} \int_{0}^{2 \pi} \ln \left|p_{n}\left(\lambda, r e^{i \theta}\right)\right| d \theta, \\
L_{n}^{+}(\lambda, w) & :=d^{-n} \sum_{j=1}^{N_{d}(n)} \ln ^{+}\left|w-w_{n, j}(\lambda)\right|, \\
L_{n}(\lambda, w) & :=d^{-n} \ln \left|p_{n}(\lambda, w)\right|,
\end{aligned}
$$

where $p_{n}(\lambda, w)=: \prod_{j=1}^{N_{d}(n)}\left(w-w_{n, j}(\lambda)\right)$ are the polynomials associated to the family $f$ by Theorem 2.1 .

The pointwise convergence of $L_{n}(\lambda, w)$ to $L$ for $|w|<1$ is quite a straightforward consequence of Theorem 2.3 and immediately implies that $d^{-n}\left[\operatorname{Per}_{n}(w)\right]$ converges to $T_{\text {bif }}$ when $|w|<1$. However, when $|w| \geq 1$ and $\lambda$ is a nonhyperbolic parameter, the control of $L_{n}(\lambda, w)=d^{-n} \sum \ln \left|w-w_{n, j}(\lambda)\right|$ is very delicate because $f_{\lambda}$ may have many cycles whose multipliers are close to $w$. This is why we introduce the PSH functions $L_{n}^{+}$, which both coincide with $L_{n}$ on the hyperbolic components and are quite easily seen to converge nicely.

\subsection{A basic result}

We present here what is obtained by combining the dynamical Theorem 2.3 with basic potential-theoretic facts. Our main result is the following.

Theorem 3.1. Let $f: M \times \mathbf{P}^{1} \rightarrow \mathbf{P}^{1}$ be a holomorphic family of degree $d \geq 2$ rational maps. Let $L(\lambda)$ be the Lyapunov exponent of $\left(\mathbf{P}^{1}, f_{\lambda}, \mu_{\lambda}\right)$, where $\mu_{\lambda}$ is the maximal entropy measure of $f_{\lambda}$. Let $\left(L_{n}\right)_{n},\left(L_{n}^{r}\right)_{n}$, and $\left(L_{n}^{+}\right)_{n}$ be the sequences of PSH functions defined as above. Then

(1) the sequence $L_{n}$ converges pointwise to $L$ on $M \times \Delta$ and, for any $w \in \Delta$, the sequence $L_{n}(\cdot, w)$ converges in $L_{\mathrm{loc}}^{1}$ to $L$ on $M$; 
(2) the sequence $L_{n}^{r}$ converges pointwise and in $L_{\text {loc }}^{1}$ to $L$ on $M$ for $r \geq 0$;

(3) the sequence $L_{n}^{+}$converges pointwise and in $L_{\mathrm{loc}}^{1}$ to $L$ on $M \times \mathbf{C}$ and, for every $w \in \mathbf{C}$, the sequence $L_{n}^{+}(\cdot, w)$ converges in $L_{\mathrm{loc}}^{1}$ to $L$ on $M$;

(4) the sequence $L_{n}$ converges in $L_{\mathrm{loc}}^{1}$ to $L$ on $M \times \mathbf{C}$.

Let us stress that Theorem 1.1 follows immediately from the first, second, and last statements of Theorem 3.1 by taking $d d^{c}$.

Proof. All the statements are local, and therefore, taking charts, we may assume that $M=\mathbf{C}^{k}$. We write the polynomials $p_{n}$ as follows:

$$
p_{n}(\lambda, w)=: \prod_{i=1}^{N_{d}(n)}\left(w-w_{n, j}(\lambda)\right) .
$$

Throughout the proof, we shall use the fact that $d^{-n} N_{d}(n) \sim 1 / n$ (see Theorem 2.1). In particular, this implies that the sequences $L_{n}$ and $L_{n}^{+}$are locally uniformly bounded from above.

- We first establish the convergence of $L_{n}(\lambda, w)$ when $|w|<1$. According to Theorem 2.1, the set $\left\{w_{n, j}(\lambda) / w_{n, j}(\lambda) \neq 1\right\}$ coincides with the set of multipliers of cycles of exact period $n$ (counted with multiplicity) from which the cycles of multiplier 1 are deleted. Using the notation $R_{n}^{*}(\lambda):=\{p \in$ $\mathbf{P}^{1} / p$ has exact period $n$ and $\left.\left|\left(f_{\lambda}^{n}\right)^{\prime}(p)\right|>1\right\}$, we thus have

$$
\sum_{j=1}^{N_{d}(n)} \ln ^{+}\left|w_{n, j}(\lambda)\right|=\frac{1}{n} \sum_{p \in R_{n}^{*}(\lambda)} \ln \left|\left(f^{n}\right)^{\prime}(p)\right| .
$$

Since $f_{\lambda}$ has a finite number of nonrepelling cycles (Fatou's theorem), one sees that there exists $n(\lambda) \in \mathbf{N}$ such that

$$
n \geq n(\lambda) \Rightarrow\left|w_{n, j}(\lambda)\right|>1, \quad \text { for any } 1 \leq j \leq N_{d}(n)
$$

By (3.1) and (3.2), one gets

$$
\begin{aligned}
L_{n}(\lambda, 0) & =d^{-n} \sum_{j=1}^{N_{d}(n)} \ln \left|w_{n, j}(\lambda)\right| \\
& =d^{-n} \sum_{j=1}^{N_{d}(n)} \ln ^{+}\left|w_{n, j}(\lambda)\right|=\frac{d^{-n}}{n} \sum_{R_{n}^{*}(\lambda)} \ln \left|\left(f^{n}\right)^{\prime}(p)\right|
\end{aligned}
$$


for $n \geq n(\lambda)$ which, by Theorem 2.3 , yields

$$
\lim _{n} L_{n}(\lambda, 0)=L(\lambda), \quad \forall \lambda \in M .
$$

Let us now pick $w \in \Delta$. By (3.2), we have $L_{n}(\lambda, w)-L_{n}(\lambda, 0)=d^{-n} \times$ $\sum_{j} \ln \left(\left|w_{n, j}(\lambda)-w\right|\right) /\left(\left|w_{n, j}(\lambda)\right|\right)$ and $\ln (1-|w|) \leq \ln \left(\left|w_{n, j}(\lambda)-w\right|\right) /$ $\left(\left|w_{n, j}(\lambda)\right|\right) \leq \ln (1+|w|)$ for $1 \leq j \leq N_{d}(n)$ and $n \geq n(\lambda)$. We thus get

$$
d^{-n} N_{d}(n) \ln (1-|w|) \leq\left|L_{n}(\lambda, w)-L_{n}(\lambda, 0)\right| \leq d^{-n} N_{d}(n) \ln (1+|w|)
$$

for $n \geq n(\lambda)$ and, using (3.3), we get $\lim _{n} L_{n}(\lambda, w)=L(\lambda)$ for any $(\lambda, w) \in$ $M \times \Delta$.

The $L_{\text {loc }}^{1}$ convergence of $L_{n}(\cdot, w)$ now follows immediately from Theorem 2.5.

- Let us show that the convergence of $L_{n}(\lambda, 0)=L_{n}^{0}$ implies the convergence of $L_{n}^{r}$ for any $r>0$. We essentially show that $\lim _{n}\left|L_{n}^{r}(\lambda)-L_{n}(\lambda, 0)\right|=0$ by using the formula $\ln \max (|a|, r)=(1 / 2 \pi) \int_{0}^{2 \pi} \ln \left|a-r e^{i \theta}\right| d \theta$. Indeed, this formula yields

$$
L_{n}^{r}(\lambda)=\frac{1}{2 \pi d^{n}} \int_{0}^{2 \pi} \ln \prod_{j}\left|r e^{i \theta}-w_{n, j}(\lambda)\right| d \theta=d^{-n} \sum_{j} \ln \max \left(\left|w_{n, j}(\lambda)\right|, r\right) .
$$

Since $\left|w_{n, j}(\lambda)\right| \geq 1$ for $n \geq n(\lambda)$ (see (3.2)), we deduce from the above identity that

$$
\begin{aligned}
L_{n}^{r}(\lambda) & =d^{-n} \sum_{j} \ln \left|w_{n, j}(\lambda)\right|+d^{-n} \sum_{1 \leq\left|w_{n, j}(\lambda)\right|<r} \ln \frac{r}{\left|w_{n, j}(\lambda)\right|} \\
& =L_{n}(\lambda, 0)+d^{-n} \sum_{1 \leq\left|w_{n, j}(\lambda)\right|<r} \ln \frac{r}{\left|w_{n, j}(\lambda)\right|},
\end{aligned}
$$

and thus

$$
0 \leq L_{n}^{r}(\lambda)-L_{n}(\lambda, 0)=d^{-n} \sum_{1 \leq\left|w_{n, j}(\lambda)\right|<r} \ln \frac{r}{\left|w_{n, j}(\lambda)\right|} \leq d^{-n} N_{d}(n) \ln ^{+} r .
$$

By (3.3), this implies that $L_{n}^{r}$ converges pointwise to $L$. It also shows that $\left(L_{n}^{r}\right)_{n}$ is locally uniformly bounded from above which, by Theorem 2.5 , implies that $\left(L_{n}^{r}\right)_{n}$ converges to $L$ in $L_{\text {loc }}^{1}(M)$.

- Let us now deal with the convergence of $L_{n}^{+}$. We will show that $L_{n}^{+}(\cdot, w)$ converges pointwise to $L$ on $M$ for every $w \in \mathbf{C}$. As $\left(L_{n}^{+}\right)_{n}$ is locally uniformly bounded, this implies the convergence of $L_{n}^{+}(\cdot, w)$ in $L_{\text {loc }}^{1}(M)$ (see 
Theorem 2.5), and the convergence of $L_{n}^{+}$in $L_{\text {loc }}^{1}(M \times \mathbf{C})$ then follows by Lebesgue's theorem.

We have to estimate $L_{n}^{+}(\lambda, w)-L_{n}(\lambda, 0)=: \epsilon_{n}(\lambda, w)$ on $M$. Let us fix $\lambda \in M, w \in \mathbf{C}$ and pick $R>|w|$. We may assume that $n \geq n(\lambda)$ so that $\left|w_{n, j}(\lambda)\right| \geq 1$ for all $1 \leq j \leq N_{d}(n)$ (see (3.2)), and we then decompose $\epsilon_{n}(\lambda, w)$ in the following way:

$$
\begin{aligned}
\epsilon_{n}(\lambda, w)= & d^{-n} \sum_{1 \leq\left|w_{n, j}(\lambda)\right|<R+1} \ln ^{+}\left|w_{n, j}(\lambda)-w\right| \\
& +d^{-n} \sum_{\left|w_{n, j}(\lambda)\right| \geq R+1} \ln \frac{\left|w_{n, j}(\lambda)-w\right|}{\left|w_{n, j}(\lambda)\right|} \\
& -d^{-n} \sum_{1 \leq\left|w_{n, j}(\lambda)\right|<R+1} \ln \left|w_{n, j}(\lambda)\right| .
\end{aligned}
$$

We may write this decomposition as $\epsilon_{n}(\lambda, w)=: \epsilon_{n, 1}(\lambda, w)+\epsilon_{n, 2}(\lambda, w)-$ $\epsilon_{n, 1}(\lambda, 0)$. As $L_{n}(\lambda, 0)$ converges to $L$, we simply have to check that $\epsilon_{n, 1}(\lambda, w)$ and $\epsilon_{n, 2}(\lambda, w)$ tend to 0 when $n$ tends to $\infty$. One clearly has $0 \leq \epsilon_{n, 1}(\lambda, w) \leq$ $d^{-n} N_{d}(n) \ln (2 R+1)$, and thus $\lim _{n} \epsilon_{n, 1}(\lambda, w)=0$. Similarly, $\lim _{n} \epsilon_{n, 2}(\lambda$, $w)=0$ follows from the fact that, for $\left|w_{n, j}(\lambda)\right|>R+1>|w|+1$, one has

$$
\begin{aligned}
\ln \left(1-\frac{R}{R+1}\right) & \leq \ln \frac{\left|w_{n, j}(\lambda)\right|-R}{\left|w_{n, j}(\lambda)\right|} \leq \ln \frac{\left|w_{n, j}(\lambda)-w\right|}{\left|w_{n, j}(\lambda)\right|} \\
& \leq \ln \frac{\left|w_{n, j}(\lambda)\right|+R}{\left|w_{n, j}(\lambda)\right|} \leq \ln \left(1+\frac{R}{R+1}\right)
\end{aligned}
$$

- We are finally ready to prove the $L_{\text {loc }}^{1}$ convergence of $\left(L_{n}\right)_{n}$. As the functions $L_{n}$ are PSH and the sequence $\left(L_{n}\right)_{n}$ is locally uniformly bounded from above, we shall again use the compacity properties of PSH functions given by Theorem 2.5. Since $L_{n}(\lambda, 0)$ converges to $L(\lambda)$, the sequence $\left(L_{n}\right)_{n}$ does not converge to $-\infty$, and it therefore suffices to show that, among PSH functions on $M \times \mathbf{C}$, the function $L$ is the only possible limit for $\left(L_{n}\right)_{n}$ in $L_{\text {loc }}^{1}(M \times \mathbf{C})$.

Let $\varphi$ be a PSH function on $M \times \mathbf{C}$, and let $\left(L_{n_{j}}\right)_{j}$ be a subsequence of $\left(L_{n}\right)_{n}$ which converges to $\varphi$ in $L_{\text {loc }}^{1}(M \times \mathbf{C})$. Pick $\left(\lambda_{0}, w_{0}\right) \in M \times \mathbf{C}$. We have to prove that $\varphi\left(\lambda_{0}, w_{0}\right)=L\left(\lambda_{0}\right)$.

Let us first observe that $\varphi\left(\lambda_{0}, w_{0}\right) \leq L\left(\lambda_{0}\right)$. Take a ball $B_{\epsilon}$ of radius $\epsilon$ and centered at $\left(\lambda_{0}, w_{0}\right) \in M \times \mathbf{C}$. By the submean value property and the 
$L_{\text {loc }}^{1}$-convergence of $L_{n}^{+}$, we have

$$
\begin{aligned}
\varphi\left(\lambda_{0}, w_{0}\right) & \leq \frac{1}{\left|B_{\epsilon}\right|} \int_{B_{\epsilon}} \varphi d m=\lim _{j} \frac{1}{\left|B_{\epsilon}\right|} \int_{B_{\epsilon}} L_{n_{j}} d m \\
& \leq \lim _{j} \frac{1}{\left|B_{\epsilon}\right|} \int_{B_{\epsilon}} L_{n_{j}}^{+} d m=\frac{1}{\left|B_{\epsilon}\right|} \int_{B_{\epsilon}} L d m,
\end{aligned}
$$

and then, making $\epsilon \rightarrow 0$, one obtains $\varphi\left(\lambda_{0}, w_{0}\right) \leq L\left(\lambda_{0}\right)$.

Let us now check that $\limsup _{j} L_{n_{j}}\left(\lambda_{0}, w_{0} e^{i \theta}\right)=L\left(\lambda_{0}\right)$ for almost all $\theta \in$ $[0,2 \pi]$. Let $r_{0}:=\left|w_{0}\right|$. As we saw, $L_{n}^{+}$converges pointwise to $L$, and therefore

$$
\underset{j}{\limsup } L_{n_{j}}\left(\lambda_{0}, w_{0} e^{i \theta}\right) \leq \underset{j}{\limsup } L_{n_{j}}^{+}\left(\lambda_{0}, w_{0} e^{i \theta}\right)=L\left(\lambda_{0}\right) .
$$

On the other hand, by pointwise convergence of $L_{n}^{r_{0}}$ to $L$ and Fatou's lemma, we have

$$
\begin{aligned}
L\left(\lambda_{0}\right) & =\lim _{n} L_{n}^{r_{0}}\left(\lambda_{0}\right)=\underset{j}{\limsup } \frac{1}{2 \pi} \int_{0}^{2 \pi} L_{n_{j}}\left(\lambda_{0}, r_{0} e^{i \theta}\right) d \theta \\
& \leq \frac{1}{2 \pi} \int_{0}^{2 \pi} \limsup _{j} L_{n_{j}}\left(\lambda_{0}, r_{0} e^{i \theta}\right) d \theta
\end{aligned}
$$

and the desired property follows immediately.

To end the proof, we argue by contradiction and assume that $\varphi\left(\lambda_{0}, w_{0}\right)<$ $L\left(\lambda_{0}\right)$. As $\varphi$ is upper semicontinuous and $L$-continuous (see Theorem 2.4), there exists a neighborhood $V_{0}$ of $\left(\lambda_{0}, w_{0}\right)$ and $\epsilon>0$ such that

$$
\varphi-L \leq-\epsilon \text { on } V_{0}
$$

Pick a small ball $B_{\lambda_{0}}$ centered at $\lambda_{0}$ and a small disc $\Delta_{w_{0}}$ centered at $w_{0}$ such that $B_{0}:=B_{\lambda_{0}} \times \Delta_{w_{0}}$ is relatively compact in $V_{0}$. Then, according to Hartogs's lemma (see [13, page 94]), we have

$$
\limsup \left(\sup _{B_{0}}\left(L_{n_{j}}-L\right)\right) \leq \sup _{B_{0}}(\varphi-L) \leq-\epsilon
$$

This is impossible since, as we have seen before, we may find $\left(\lambda_{0}, r_{0} e^{i \theta_{0}}\right) \in B_{0}$ such that $\lim \sup _{j}\left(L_{n_{j}}\left(\lambda_{0}, r_{0} e^{i \theta_{0}}\right)-L\left(\lambda_{0}\right)\right)=0$.

REMARK 3.2. Using standard techniques, one may deduce from the fourth assertion of Theorem 3.1 that the set of multipliers $w$ for which the bifurcation current $T_{\text {bif }}$ is not a limit of the sequence $d^{-n}\left[\operatorname{Per}_{n}(w)\right]$ is contained in a polar subset of the complex plane. 


\subsection{Further results}

The fact that the functions $L_{n}^{+}$and $L_{n}$ coincide on hyperbolic components would easily yield the convergence of $d^{-n}\left[\operatorname{Per}_{n}(w)\right]$ towards $T_{\text {bif }}$ for any $w \in \mathbf{C}$ if the density of hyperbolic parameters in $M$ were known.

One may, however, overcome this difficulty when the hyperbolic parameters are sufficiently nicely distributed. Here we establish a few facts of this nature which we use in our study of polynomial families in Section 4.

The following proposition summarizes some useful remarks.

Proposition 3.3. Let us make the same assumptions and adopt the same notations as in Theorem 3.1. Let $w_{0} \in \mathbf{C}$. Then the following hold.

(1) Any PSH limit value of $L_{n}\left(\lambda, w_{0}\right)$ in $L_{\mathrm{loc}}^{1}(M)$ is smaller than $L$.

(2) If a subsequence $L_{n_{k}}\left(\lambda, w_{0}\right)$ converges pointwise to $L$ on the stable set, then it also converges to $L$ in $L_{\text {loc }}^{1}(M)$.

(3) Assume that $\left|w_{0}\right|=1$ and that the family has no persistent neutral cycle. If a subsequence $L_{n_{k}}\left(\lambda, w_{0}\right)$ converges to $\varphi$ in $L_{\mathrm{loc}}^{1}(M)$, then $\varphi$ is pluriharmonic on any stable component $\Omega$ and the convergence is locally uniform on $\Omega$.

(4) For any hyperbolic component $\Omega \subset M$, the sequence $L_{n}\left(\lambda, w_{0}\right)$ converges locally uniformly to $L$ on $\Omega$.

Proof. (1) Let us set $\varphi_{n}(\lambda):=L_{n}\left(\lambda, w_{0}\right)$ and assume that a subsequence $\varphi_{n_{j}}$ converges in $L_{\text {loc }}^{1}(M)$ to some PSH function $\varphi$. Since $L_{n}^{+}\left(\lambda, w_{0}\right)$ converges to $L$ in $L_{\text {loc }}^{1}(M)$ and since $\varphi_{n_{j}}(\lambda) \leq \mathrm{七}_{n_{j}}^{+}\left(\lambda, w_{0}\right)$, we get $\varphi\left(\lambda_{0}\right) \leq$ $\left(1 /\left|B_{\epsilon}\right|\right) \int_{B_{\epsilon}} \varphi d m \leq\left(1 /\left|B_{\epsilon}\right|\right) \int_{B_{\epsilon}} L d m$ for any small ball $B_{\epsilon}$ centered at $\lambda_{0}$. The desired inequality then follows by making $\epsilon \rightarrow 0$.

(2) Recall that the stable set is an open dense subset of $M$. Let $\varphi$ be any PSH limit of $L_{n_{k}}\left(\lambda, w_{0}\right)$ in $L_{\text {loc }}^{1}(M)$. We have to show that $\varphi=L$. By the first assertion, $\varphi \leq L$. As $\varphi=L$ on a dense subset, the semicontinuity of $\varphi$ and the continuity of $L$ (see Theorem 2.4) imply that $\varphi \geq L$.

(3) Using Remark 2.2, one sees that the functions $L_{n_{k}}\left(\lambda, w_{0}\right)$ are pluriharmonic on $\Omega$. This implies that $\varphi$ itself is pluriharmonic on $\Omega$ and that $L_{n_{k}}\left(\lambda, w_{0}\right)$ converges actually locally uniformly on $\Omega$ to $\varphi$.

(4) If $\lambda$ is a hyperbolic parameter, then $f_{\lambda}$ has only attracting or repelling cycles and is expansive on its Julia set. Thus, as $f_{\lambda}$ has at most a finite number of attracting cycles, one sees that $\left|w_{n, j}(\lambda)\right| \geq\left|w_{0}\right|+1$ for all $1 \leq$ $j \leq N_{d}(n)$ and $n$ big enough. In other words, $L_{n}\left(\lambda, w_{0}\right)=L_{n}^{+}\left(\lambda, w_{0}\right)$ for $n$ big enough, and therefore, according to Theorem $3.1, L_{n}\left(\lambda, w_{0}\right)$ converges 
to $L(\lambda)$. By Theorem $2.5, L_{n}\left(\lambda, w_{0}\right)$ converges to $L$ in $L_{\text {loc }}^{1}(\Omega)$. The local uniform convergence then follows from the previous assertion.

In the last section of the paper, we will focus on the case $\left|w_{0}\right|=1$ and work with polynomial families. Slicing the parameter space in different ways, we will obtain 1-dimensional holomorphic families where the hyperbolic parameters are well distributed. Here is a typical example which, in particular, covers the case of the quadratic polynomial family.

Proposition 3.4. Let $M$ be a Riemann surface, and let $f: M \times \mathbf{P}^{1} \rightarrow \mathbf{P}^{1}$ be a holomorphic family of degree $d \geq 2$ rational maps which satisfies the following two conditions:

(1) the bifurcation locus is contained in the closure of hyperbolic parameters;

(2) the set of nonhyperbolic parameters is compact in $M$.

Let $L(\lambda)$ and $L_{n}(\lambda, w)$ be the subharmonic functions defined in Theorem 3.1. Then, if $\left|w_{0}\right|=1$, the sequence $L_{n}\left(\lambda, w_{0}\right)$ converges to $L$ in $L_{\text {loc }}^{1}(M)$.

The proof of this proposition is based on the following technical lemma. This lemma actually deals with more general situations which we will encounter in Section 4.

Lemma 3.5. Let $M$ be a Riemann surface, and let $f: M \times \mathbf{P}^{1} \rightarrow \mathbf{P}^{1}$ be a holomorphic family of degree $d \geq 2$ rational maps. Let $L(\lambda)$ and $L_{n}(\lambda, w)$ be the subharmonic functions defined in Theorem 3.1. Let $w_{0} \in \mathbf{C}$ with $\left|w_{0}\right|=1$, and let $\varphi$ be a subharmonic limit value of $L_{n}\left(\lambda, w_{0}\right)$ in $L_{\mathrm{loc}}^{1}(M)$ such that

(1) the bifurcation locus is contained in the closure of the set of parameters where $\varphi=L$;

(2) $\varphi=L$ on the stable component which is not relatively compact in $M$.

Then $\varphi=L$.

Proof. We start by proving the lemma. On several occasions we shall use the fact that the function $L$ is continuous (see Theorem 2.4). Assume that $\varphi_{n_{j}}:=L_{n}\left(\cdot, w_{0}\right)$ converges to $\varphi$. Then the holomorphic functions $p_{n_{j}}\left(\lambda, w_{0}\right)$ cannot vanish identically for $j$ big enough. According to Remark 2.2, this implies that the functions $\varphi_{n_{j}}$ are harmonic on all stable components of $M$. This leads to the simple but crucial observation that $\varphi$ is harmonic on any stable component, or in other words, that the Laplacian $\Delta \varphi$ is supported in the bifurcation locus.

According to the first assertion of Proposition 3.3, we have $\varphi \leq L$. We may now see that $\varphi=L$ on the bifurcation locus. Indeed, if $\lambda_{0}$ belongs to 
the bifurcation locus, then, by assumption, there exists a sequence $\lambda_{k}$ which converges to $\lambda_{0}$ such that $\varphi\left(\lambda_{k}\right)=L\left(\lambda_{k}\right)$. Then, using the upper semicontinuity of $\varphi$ and the continuity of $L$, we get $\varphi\left(\lambda_{0}\right)=\limsup _{\lambda \rightarrow \lambda_{0}} \varphi(\lambda) \geq$ $\lim \sup \varphi\left(\lambda_{k}\right)=\lim L\left(\lambda_{k}\right)=L\left(\lambda_{0}\right)$.

By the first observation and the fact that $L$ is continuous, we see that $\varphi$ is continuous on the support of its Laplacian. According to some well-known continuity principle, this implies that $\varphi$ is continuous on $M$. We may now prove that $\varphi \equiv L$. If this were not the case, then $\varphi\left(\lambda_{0}\right)<L\left(\lambda_{0}\right)$ for some $\lambda_{0} \in M$. As $L$ and $\varphi$ coincide on the bifurcation locus and (by assumption) on nonrelatively compact stable components, $\lambda_{0}$ would belong to some stable component $\Omega$ which is relatively compact in $M$. This contradicts the maximum principle since $(\varphi-L)$ is continuous on $\bar{\Omega}$, harmonic on $\Omega$, and vanishes on $b \Omega$.

Let us now establish the proposition. By the fourth assertion of Proposition 3.3 , the sequence $L_{n}\left(\lambda, w_{0}\right)$ does not converge to $-\infty$. According to Theorem 2.5, it thus suffices to show that any subharmonic limit value $\varphi$ of $L_{n}\left(\lambda, w_{0}\right)$ in $L_{\text {loc }}^{1}(M)$ coincides with $L$. This follows immediately from Lemma 3.5 since, once again by the fourth assertion of Proposition 3.3, $\varphi=L$ on the nonrelatively compact stable components.

REMARK 3.6. The first assumption of Proposition 3.4 is a well-known open question in the space of polynomials of degree $d \geq 3$. This explains why a slicing argument will be used in the proof of Theorem 1.2.

\section{§4. Distribution of $\operatorname{Per}_{n}(w)$ in polynomial families}

\subsection{The space of degree $d$ polynomials}

Let $\mathcal{P}_{d}$ be the space of polynomials of degree $d \geq 2$ with $d-1$ marked critical points up to conjugacy by affine transformations. Although this space has a natural structure of affine variety of dimension $d-1$, we shall actually work with a specific parameterization of $\mathcal{P}_{d}$ which was introduced by Dujardin and Favre in [12]. We refer to their paper and to the seminal paper of Branner and Hubbard [5] for a better description of $\mathcal{P}_{d}$.

For every $(c, a):=\left(c_{1}, c_{2}, \ldots, c_{d-2}, a\right) \in \mathbf{C}^{d-1}$, we denote by $P_{c, a}$ the polynomial of degree $d$ whose critical points are $\left(0, c_{1}, \ldots, c_{d-2}\right)$ and such that $P_{c, a}(0)=a^{d}$. This polynomial is explicitly given by

$$
P_{c, a}:=\frac{1}{d} z^{d}+\sum_{2}^{d-1} \frac{(-1)^{d-j}}{j} \sigma_{d-j}(c) z^{j}+a^{d},
$$


where $\sigma_{i}(c)$ is the symmetric polynomial of degree $i$ in $\left(c_{1}, \ldots, c_{d-2}\right)$. For convenience, we shall set $c_{0}:=0$.

We shall thus work within the holomorphic family $\left(P_{c, a}\right)_{(c, a) \in M}$, where the parameter space $M$ is simply $\mathbf{C}^{d-1}$. As explained by Milnor [16], it is convenient to consider the projective compactification $\mathbf{P}^{d-1}$ of $\mathbf{C}^{d-1}=M$ and see the sets $\operatorname{Per}_{n}(w)$ as algebraic hypersurfaces of $\mathbf{P}^{d-1}$. We shall denote the projective space at infinity $\left\{[c: a: 0] ;(c, a) \in \mathbf{C}^{d-1} \backslash\{0\}\right\}$ by $\mathbf{P}_{\infty}$.

\subsection{The behavior of the bifurcation locus at infinity}

We aim to show that the bifurcation locus of the family $\left\{P_{c, a}\right\}_{(c, a) \in \mathbf{C}^{d-1}}$ can only cluster on certain hypersurfaces of $\mathbf{P}_{\infty}$. The ideas here are essentially those used by Branner and Hubbard for proving the compactness of the connectedness locus (see [5, Chapter 1, Section 3]), but we also borrow from Dujardin and Favre [12].

For every $0 \leq i \leq d-2$, we will denote by $\alpha_{i}$ the homogeneous polynomial defined by

$$
\alpha_{i}(c, a):=P_{c, a}\left(c_{i}\right)=\frac{1}{d} c_{i}^{d}+\sum_{j=2}^{d-1} \frac{(-1)^{d-j}}{j} \sigma_{d-j}(c) c_{i}^{j}+a^{d},
$$

and we will consider the hypersurface $\Gamma_{i}$ of $\mathbf{P}_{\infty}$ defined by

$$
\Gamma_{i}:=\left\{[c: a: 0] / \alpha_{i}(c, a)=0\right\}
$$

By a simple degree argument, one sees that $P_{c, a}(0)=P_{c, a}\left(c_{1}\right)=\cdots=$ $P_{c, a}\left(c_{d-2}\right)=0$ implies that $c_{1}=\cdots=c_{d-2}=a=0$. This observation and Bezout's theorem lead to the following.

Lemma 4.1. The intersection $\Gamma_{0} \cap \Gamma_{1} \cap \cdots \cap \Gamma_{d-2}$ is empty, and $\Gamma_{i_{1}} \cap$ $\cdots \cap \Gamma_{i_{k}}$ has codimension $k$ in $\mathbf{P}_{\infty}$ if $0 \leq i_{1}<\cdots<i_{k} \leq d-2$.

We shall denote by $\mathcal{P}_{i}$ the set of parameters $(c, a)$ for which the critical point $c_{i}$ of $P_{c, a}$ has a bounded forward orbit (recall that $c_{0}=0$ ). The announced result can now be stated as follows.

Theorem 4.2. For every $0 \leq i \leq d-2$, the cluster set of $\mathcal{P}_{i}$ in $\mathbf{P}_{\infty}$ is contained in $\Gamma_{i}$ and, in particular, the connectedness locus is compact in $\mathbf{C}^{d-1}$.

Since any cycle of attracting basins captures a critical orbit, the above theorem implies that the intersection of $\mathbf{P}_{\infty}$ with an algebraic subset of the 
form $\operatorname{Per}_{m_{1}}\left(\eta_{1}\right) \cap \cdots \cap \operatorname{Per}_{m_{k}}\left(\eta_{k}\right)$ is contained in some $\Gamma_{i_{1}} \cap \cdots \cap \Gamma_{i_{k}}$ as soon as the $m_{i}$ are mutually distinct and the $\left|\eta_{i}\right|$ strictly smaller than 1 . Then, using Bezout's theorem, one gets the following.

Corollary 4.3. If $1 \leq k \leq d-1, m_{1}<m_{2}<\cdots<m_{k}$, and $\sup _{1 \leq i \leq k}\left|\eta_{i}\right|<$ 1 , then $\operatorname{Per}_{m_{1}}\left(\eta_{1}\right) \cap \cdots \cap \operatorname{Per}_{m_{k}}\left(\eta_{k}\right)$ is an algebraic subset of codimension $k$ whose intersection with $\mathbf{C}^{d-1}$ is not empty.

The proof of Theorem 4.2 relies on estimates on the Green function and, more precisely, on the following result which is proved in [12, Section 6.1].

Proposition 4.4. Let $g_{c, a}(z):=\lim _{n} d^{-n} \ln ^{+}\left|P_{c, a}^{n}(z)\right|$ be the Green function of $P_{c, a}$, and let $G$ be the function defined on $\mathbf{C}^{d-1}$ by: $G(c, a):=$ $\max \left\{g_{c, a}\left(c_{k}\right) ; 0 \leq k \leq d-2\right\}$. Let $\delta:=\left(\sum_{k=0}^{d-2} c_{k}\right) /(d-1)$. Then the following estimates occur:

(1) $\max \left\{g_{c, a}(z), G(c, a)\right\} \geq \ln |z-\delta|-\ln 4$;

(2) $G(c, a)=\ln ^{+} \max \left\{|a|,\left|c_{k}\right|\right\}+O(1)$.

Proof of Theorem 4.2. Let $\|(c, a)\|:=\max \left(|a|,\left|c_{k}\right|\right)$. We simply have to check that $\alpha_{i}((c, a) /\|(c, a)\|)$ tends to 0 when $\|(c, a)\|$ tends to $+\infty$ and that $g_{c, a}\left(c_{i}\right)$ stays equal to 0 . As $P_{c, a}\left(c_{i}\right)=\alpha_{i}(c, a)$ and $g_{c, a}\left(c_{i}\right)=0$, the estimates given by Proposition 4.4 yield

$$
\begin{aligned}
\ln ^{+}\|(c, a)\|+O(1) & =\max \left(d g_{c, a}\left(c_{i}\right), G(c, a)\right)=\max \left(g_{c, a} \circ P_{c, a}\left(c_{i}\right), G(c, a)\right) \\
& \geq \ln \frac{1}{4}\left|\alpha_{i}(c, a)-\delta\right| .
\end{aligned}
$$

Since $\alpha_{i}$ is $d$-homogeneous, we then get for $\|(c, a)\|>1$

$$
(1-d) \ln \|(c, a)\|+O(1) \geq \ln \frac{1}{4}\left|\alpha_{i}\left(\frac{(c, a)}{\|(c, a)\|}\right)-\frac{\delta}{\|(c, a)\|^{d}}\right|,
$$

and the conclusion follows since $\delta /\|(c, a)\|^{d}$ tends to 0 when $\|(c, a)\|$ tends to $+\infty$.

\subsection{Proof of the main result}

We shall denote by $\lambda$ the parameter in $\mathbf{C}^{d-1}$ (i.e., $\lambda:=(c, a)$ ), and we will then set

$$
\varphi_{n}(\lambda):=d^{-n} \ln \left|p_{n}(\lambda, w)\right|,
$$

where the polynomials $p_{n}(\lambda, w)$ are those given by Theorem 2.1. We have to show that the sequence $\left(\varphi_{n}\right)_{n}$ converges to $L$ in $L_{\text {loc }}^{1}$. When $|w|<1$, this 
has been shown to be true for any holomorphic family of rational maps (see the first assertion of Theorem 3.1), so we assume that $|w|=1$.

As it has been previously observed, the case $d=2$ is covered by Proposition 3.4. To give a flavor of the proof when $d \geq 2$, we will first sketch it for $d=3$.

Sketch of proof for degree 3 polynomials. Let us first treat the problem on a curve $\operatorname{Per}_{k_{0}}\left(\eta_{0}\right)$ for $\left|\eta_{0}\right|<1$. We will show that the sequence $\varphi_{n}(\lambda)$ converges uniformly to $L$ near any stable (in $\operatorname{Per}_{k_{0}}\left(\eta_{0}\right)$ ) parameter $\lambda_{0}$. For this purpose, one desingularizes an irreducible component of $\operatorname{Per}_{k_{0}}\left(\eta_{0}\right)$ containing $\lambda_{0}$ and thus obtains a 1-dimensional holomorphic family $\left(P_{\pi(u)}\right)_{u \in M}$. Keeping in mind that the elements of this family are degree 3 polynomials which do admit an attracting basin of period $k_{0}$ and using the fact that the connectedness locus in $\mathbf{C}^{2}$ is compact, one sees that the family $\left(P_{\pi(u)}\right)_{u \in M}$ satisfies the assumptions of Proposition 3.4. The associated sequence $L_{n}(u, w)=\varphi_{n}(\pi(u))$ converges therefore in $L_{\text {loc }}^{1}$ to $L$, and this convergence is locally uniform on stable components by Proposition 3.3.

Let us now consider the problem on the full parameter space $\mathbf{C}^{2}$. Since the family $\left\{P_{c, a}\right\}_{(c, a) \in \mathbf{C}^{2}}$ contains hyperbolic parameters, the fourth assertion of Proposition 3.3 shows that the sequence $\varphi_{n}(\lambda)$ does not converge to $-\infty$. According to Theorem 2.5, it thus suffices to show that any PSH limit value $\varphi$ of $\varphi_{n}(\lambda)$ in $L_{\text {loc }}^{1}\left(\mathbf{C}^{2}\right)$ coincides with $L$. Let us therefore assume that $\varphi_{n_{k}}$ tends to $\varphi$ in $L_{\text {loc }}^{1}\left(\mathbf{C}^{2}\right)$.

We first show that $\varphi=L$ on any open subset of the type

$$
A_{k_{0}}:=\bigcup_{|\eta|<1} \operatorname{Per}_{k_{0}}(\eta)
$$

According to the second assertion of Proposition 3.3, it suffices to show that $\varphi=L$ on any stable component $\Omega$ of $A_{k_{0}}$. By the third assertion of Proposition 3.3, $\varphi_{n_{k}}$ actually converges pointwise to $\varphi$ on $\Omega$. As (by the previous step) $\varphi_{n}(\lambda)$ converges locally uniformly on the stable components of $\operatorname{Per}_{k_{0}}(\eta)$, one thus obtains that $\varphi=L$ on $\Omega$.

According to Theorem 4.2, the set of nonhyperbolic parameters in $\mathbf{C}^{2}$ can only cluster on a finite subset of $\mathbf{P}_{\infty}$. We may therefore foliate $\mathbf{C}^{2}$ by parallel complex lines $\left(T_{t}\right)_{t \in \mathbf{C}}$ whose intersection with the set of nonhyperbolic parameters is compact. After taking a subsequence, we may assume that $\varphi_{n_{k}}$ converges to $\varphi$ in $L_{\text {loc }}^{1}\left(T_{t}\right)$ for almost every $t \in \mathbf{C}$. To conclude, it remains to 
see that $\left.\left.\varphi\right|_{T_{t}} \equiv L\right|_{T_{t}}$ for these $t$. For this, one uses Lemma 3.5. The assumptions of the lemma are satisfied since, by construction, the unbounded stable component of $T_{t}$ is hyperbolic, and the bifurcation locus in $T_{t}$ is accumulated by sets of the form $T_{t} \cap A_{k_{0}}$ where, as we have previously shown, $\varphi=L$.

Proof of Theorem 1.2. For $1 \leq q \leq d-2$, the notation $W_{q}$ will refer to any irreducible component of a $q$-codimensional analytic subspace of $\mathbf{C}^{d-1}$ of the form $\operatorname{Per}_{n_{1}}\left(\eta_{1}\right) \cap \cdots \cap \operatorname{Per}_{n_{q}}\left(\eta_{q}\right)$, where $\left(\eta_{1}, \ldots, \eta_{q}\right) \in \Delta^{q}$ and where the integers $n_{j} \geq 2$ are mutually distinct (by Corollary 4.3, such sets do exist). Let us stress that if $\lambda \in W_{q}$, then the polynomial $P_{\lambda}$ admits $q$ distinct attracting basins besides the basin at infinity. Analogously, we shall set $W_{0}:=\mathbf{C}^{d-1}$. By $W_{q}^{\text {reg }}$ we shall denote the regular part of $W_{q}$. The proof will consist in showing by decreasing induction on $0 \leq q \leq d-2$ that

$$
\left(*_{q}\right) \text { : the sequence }\left.\varphi_{n}\right|_{W_{q}} \text { tends to } L \text { in } L_{\text {loc }}^{1}\left(W_{q}^{\text {reg }}\right) \text { for any } W_{q} \text {. }
$$

Let us first establish $\left(*_{d-2}\right)$. The analytic set $W_{d-2}$ is a curve in $\mathbf{C}^{d-1}$. Desingularizing, we get a proper holomorphic map $\pi: M \rightarrow W_{d-2}$, where $M$ is a Riemann surface. We claim that the 1-dimensional holomorphic family $\left(P_{\pi(u)}\right)_{u \in M}$ satisfies the assumptions of Proposition 3.4. To see this, we observe that there exists at most one critical point of the polynomial $P_{\pi(u)}$ whose orbit is not captured by one of the $d-2$ distinct attracting basins of $P_{\pi(u)}$. Let us denote by $c(u)$ this critical point. Assume that $u_{0}$ lies in the bifurcation locus of $\left(P_{\pi(u)}\right)_{u \in M}$. Since all critical points of $P_{\pi(u)}$, except a priori $c(u)$, stay in some attracting basin for $u$ close to $u_{0}$, the orbit of $c(u)$ cannot be uniformly bounded on a small neighborhood of $u_{0}$. This implies that $c(u)$ must belong to the basin of infinity for a convenient small perturbation of $u_{0}$, and shows that $P_{\pi\left(u_{0}\right)}$ becomes hyperbolic after a convenient small perturbation. In other words, the bifurcation locus of $\left(P_{\pi(u)}\right)_{u \in M}$ is accumulated by hyperbolic parameters.

The above argument also shows that if $P_{\pi(u)}$ is nonhyperbolic, then $c(u)$ cannot belong to the basin at infinity, and therefore $P_{\pi(u)}$ belongs to the connectedness locus. Using the compactness of the connectedness locus and the properness of the map $\pi$, one sees that the set of nonhyperbolic parameters of $M$ is compact.

By Proposition 3.4, $\varphi_{n}(\pi(u))$ converges in $L_{\text {loc }}^{1}(M)$ to $L \circ \pi$. By the third assertion of Proposition 3.3, the convergence is actually pointwise on the stable components of $M$, and thus $\varphi_{n}$ converges pointwise to $L$ on the stable 
set of $W_{q}^{\text {reg }}$. By the second assertion of Proposition 3.3, $\left.\varphi_{n}\right|_{W_{q}}$ converges to $L$ in $L_{\text {loc }}^{1}\left(W_{q}^{\text {reg }}\right)$. We have proved $\left(*_{d-2}\right)$.

Assuming now that $\left(*_{q+1}\right)$ is satisfied, we shall prove that $\left(*_{q}\right)$ is true. Let us fix an irreducible $q$-codimensional analytic set $W_{q} \subset \operatorname{Per}_{n_{1}}\left(\eta_{1}\right) \cap \cdots \cap$ $\operatorname{Per}_{n_{q}}\left(\eta_{q}\right)$.

One easily deduces from Corollary 4.3 that $W_{q}$ contains hyperbolic para-

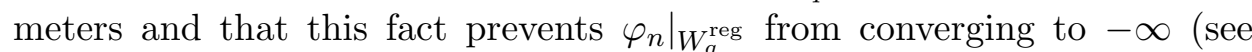
Proposition 3.3). According to Theorem 2.5, we thus have to show that for any subsequence $\left.\varphi_{n_{k}}\right|_{W_{q}^{\text {reg }}}$ which converges to some $\mathrm{PSH}$ function $\varphi$ in $L_{\text {loc }}^{1}\left(W_{q}^{\text {reg }}\right)$, one actually has $\varphi=\left.L\right|_{W_{q}^{\text {reg }} \text {. }}$

We shall use the following two facts which will be proved later.

FACT 1. Let $A_{m}$ be an open subset of $\mathbf{C}^{d-1}$ defined by

$$
A_{m}:=\bigcup_{|\eta|<1} \operatorname{Per}_{m}(\eta)
$$

where $m>\max \left(n_{1}, \ldots, n_{q}\right)$. If $W_{q}^{\mathrm{reg}} \cap A_{m}$ is not empty, then $\varphi=L$ on $W_{q}^{\text {reg }} \cap A_{m}$.

FACT 2. There exists a foliation $\bigcup_{t \in A} T_{t}$ of $\mathbf{C}^{d-1}$ by $(q+1)$-dimensional parallel affine subspaces such that, for almost every $t \in A$, the slices $T_{t} \cap$ $W_{q}$ are curves on which the set of nonhyperbolic parameters is relatively compact.

Let us consider the curves $T_{t} \cap W_{q}$ which are given by Fact 2 . By standard arguments, $\varphi_{n_{k}}$ converges to $\varphi$ in $L_{\text {loc }}^{1}$ on almost all these curves and it thus remains to show that $\varphi=L$ on them. For this purpose, we consider an irreducible component $\Gamma$ of $T_{t} \cap W_{q}$ and desingularize it. This yields a proper holomorphic map $\pi: M \rightarrow \Gamma$, where $M$ is a Riemann surface. We shall reach the conclusion by applying Lemma 3.5 to the family $\left(P_{\pi(u)}\right)_{u \in M}$.

By the properness of $\pi$ and Fact 2, the set of nonhyperbolic parameters in $M$ is compact, and therefore any nonrelatively compact stable component in $M$ is hyperbolic. Then, by the fourth assertion of Proposition 3.3, $\varphi \circ \pi=$ $L \circ \pi$ on such components.

Using Fact 1, we shall now prove that the bifurcation locus of $\left(P_{\pi(u)}\right)_{u \in M}$ is accumulated by parameters, where $\varphi \circ \pi=L \circ \pi$. Let $u_{0}$ be a point in the bifurcation locus. We may assume that $\pi$ is locally biholomorphic at $u_{0}$, and it thus suffices to accumulate $\pi\left(u_{0}\right)$ by points where $\varphi=L$. As it is well known, $u_{0}$ is accumulated by parameters $u_{k}$ such that $P_{\pi\left(u_{k}\right)} \in$ 
$\operatorname{Per}_{m_{k}}(0)$ and $m_{k} \rightarrow+\infty$ (this follows also from the general fact that $T_{\text {bif }}=$ $\left.\lim _{m} d^{-m}\left[\operatorname{Per}_{m}(0)\right]\right)$. This implies that $\pi\left(u_{0}\right)$ is accumulated by open sets of the form $W_{q} \cap A_{m_{k}}$. It then follows from Fact 1 that $\pi\left(u_{0}\right)$ is accumulated by points $\lambda_{k}$ for which $\varphi\left(\lambda_{k}\right)=L\left(\lambda_{k}\right)$. This ends the proof.

Let us finally establish the facts.

Fact 1 . Let $\Omega$ be a stable component of $W_{q}^{\text {reg }} \cap A_{m}$. According to the first and third assertions of Proposition 3.3, the sequence $\varphi_{n_{k}}-L$ converges locally uniformly to the pluriharmonic negative function $\varphi-L$ on $\Omega$ (as previously observed, $W_{q}$ contains hyperbolic parameters and therefore has no persistent neutral cycles). For all but a finite number of $\eta \in \Delta$, the analytic set $W_{q} \cap \operatorname{Per}_{m}(\eta)$ is of codimension $q+1$ (otherwise $W_{q}$ would be contained in infinitely many hypersurfaces $\operatorname{Per}_{m}(\eta)$ and $P_{\lambda}$ would have an infinite number of attracting basins when $\left.\lambda \in W_{q}\right)$. Let us thus pick $\eta_{0} \in \Delta$ and $\lambda_{0} \in \Omega \cap \operatorname{Per}_{m}\left(\eta_{0}\right)$ such that $W_{q} \cap \operatorname{Per}_{m}\left(\eta_{0}\right)$ has codimension $q+1$ and is regular at $\lambda_{0}$. Let us denote by $W_{q+1}$ the irreducible component of $W_{q} \cap \operatorname{Per}_{m}\left(\eta_{0}\right)$ to which belongs $\lambda_{0}$. Then by construction, $\lambda_{0}$ belongs to some stable component $\omega$ of $W_{q+1}^{\text {reg }}$. Combining the induction assumption $\left(*_{q+1}\right)$ with the third assertion of Proposition 3.3 , one sees that $\varphi-L=0$ on $\omega$. In particular, $\varphi\left(\lambda_{0}\right)-L\left(\lambda_{0}\right)=0$ and, by the maximum principle, $\varphi-L=0$ on $\Omega$.

It now follows from the second assertion of Proposition 3.3 that $\varphi=L$ on $W_{q}^{\text {reg }} \cap A_{m}$. Fact 1 is proved.

Fact 2. Let $\widetilde{W}_{q}$ be the algebraic subset of $\mathbf{P}^{d-1}$ such that $\widetilde{W}_{q} \cap \mathbf{C}^{d-1}=W_{q}$. When $q>0$ and $\lambda \in W_{q}$, then $P_{\lambda}$ has $q$ distinct attracting basins, and therefore at least $q$ of its critical points have a bounded orbit. According to Theorem 4.2, we thus have

$$
\widetilde{W_{q}} \cap \mathbf{P}_{\infty} \subset \underset{0 \leq i_{1}<\cdots<i_{q} \leq d-2}{\bigcup} \Gamma_{i_{1}} \cap \cdots \cap \Gamma_{i_{q}}
$$

and moreover, $\bigcup_{0 \leq i_{1}<\cdots<i_{q+1} \leq d-2} \Gamma_{i_{1}} \cap \cdots \cap \Gamma_{i_{q+1}}$ is a $(d-3-q)$-dimensional algebraic subset of $\mathbf{P}_{\infty}$. Thus, as it is classical (see [7, subchapter 7.3]), we may pick a $q$-dimensional complex plane $C_{\infty}$ in $\mathbf{P}_{\infty}$ (a point when $\left.q=0\right)$ such that

$$
C_{\infty} \cap\left(\bigcup_{0 \leq i_{1}<\cdots<i_{q+1} \leq d-2} \Gamma_{i_{1}} \cap \cdots \cap \Gamma_{i_{q+1}}\right)=\emptyset .
$$


We now slice $\mathbf{C}^{d-1}$ by $(q+1)$-dimensional parallel affine subspaces $T_{t}$ which cluster on $C_{\infty}$ in $\mathbf{P}^{d-1}$, and we write $\mathbf{C}^{d-1}=\bigcup_{t \in A} T_{t}$, where $A$ is a $(d-q-2)$ dimensional complex plane which is transverse to the foliation.

If $\lambda \in W_{q}$, then at least $q$ of the critical points of $P_{\lambda}$ belong to some attracting basin. This implies that the set of nonhyperbolic parameters in $W_{q} \cap T_{t}$ may only cluster on the intersection of $C_{\infty}$ with $\bigcup_{0 \leq i_{1}<\cdots<i_{q+1} \leq d-2} \Gamma_{i_{1}} \cap$ $\cdots \cap \Gamma_{i_{q+1}}$. The choice of $C_{\infty}$ guarantees therefore that, for all $t \in A$, the set of nonhyperbolic parameters in $W_{q} \cap T_{t}$ is compact.

It remains to show that, for almost all $t \in A$, the analytic set $W_{q} \cap T_{t}$ is a curve. For this purpose, let us denote by $\sigma: W_{q} \rightarrow A$ the canonical projection from $W_{q}$ onto $A$. The fiber of $\sigma^{-1}(a)$ has dimension greater than $(d-1)-\operatorname{dim} A-q=1$. Then, the set of points $a \in A$ for which the fiber $\sigma^{-1}\{a\}$ is of dimension strictly greater than 1 is contained in a countable union of analytic subsets of $A$ whose dimensions are smaller than $\operatorname{dim} W_{q}-$ $2=(d-1)-q-2=\operatorname{dim} A-1$ (see [7, subchapter 3.8]). It is therefore Lebesgue negligible. In other words, $W_{q} \cap T_{t}$ is a curve for almost all $t$, and Fact 2 is proved.

\section{REFERENCES}

[1] G. Bassanelli and F. Berteloot, Bifurcation currents in holomorphic dynamics on $\mathbf{P}^{k}$, J. Reine Angew. Math. 608 (2007), 201-235.

[2] - Lyapunov exponents, bifurcation currents, and laminations in bifurcation loci, Math. Ann. 345 (2009), 1-23.

[3] F. Berteloot, Lyapunov exponent of a rational map and multipliers of repelling cycles, to appear in Riv. Mat. Univ. Parma.

[4] F. Berteloot, C. Dupont, and L. Molino, Normalization of random families of holomorphic contractions and applications to dynamics, Ann. Inst. Fourier (Grenoble) 58 (2008), 2137-2168.

[5] B. Branner and J. H. Hubbard, The iteration of cubic polynomials, I: The global topology of parameter space, Acta Math. 160 (1988), 143-206.

[6] X. Buff and A. Epstein, "Bifurcation measure and postcritically finite rational maps" in Complex Dynamics: Families and Friends, A. K. Peters, Wellesley, Mass., 2009, 491-512.

[7] E. M. Chirka, Complex Analytic Sets, Kluwer Academic Publishers, 1989.

[8] L. DeMarco, Dynamics of rational maps: A current on the bifurcation locus, Math. Res. Lett. 8 (2001), 57-66.

[9] - Dynamics of rational maps: Lyapunov exponents, bifurcations, and capacity, Math. Ann. 326 (2003), 43-73.

[10] T. C. Dinh and N. Sibony, Dynamics in several complex variables: Endomorphisms of projective spaces and polynomial-like mappings, preprint, arXiv:0810.0811

[11] R. Dujardin, "Cubic polynomials: A measurable view on the parameter space" in Complex Dynamics: Families and Friends, A. K. Peters, Wellesley, Mass., 2009, 451489 . 
[12] R. Dujardin and C. Favre, Distribution of rational maps with a preperiodic critical point, Amer. J. Math. 130 (2008), 979-1032.

[13] L. Hörmander, The Analysis of Linear Partial Differential Operators, I, Springer, New York, 1983.

[14] R. Mañé, "The Hausdorff dimension of invariant probabilities of rational maps" in Dynamical Systems (Valparaiso, Chile, 1986), Lect. Notes in Math. 1331 (1988), 86-117.

[15] R. Mañé, P. Sad, and D. Sullivan, On the dynamics of rational maps, Ann. Sci. Éc. Norm. Supér. (4) (1983), 193-217.

[16] J. W. Milnor, Geometry and dynamics of quadratic rational maps, Experiment. Math. 2 (1993), 37-83.

[17] J. H. Silverman, The Arithmetic of Dynamical Systems, Grad. Texts in Math. 241, Springer, New York, 2007.

Giovanni Bassanelli

(deceased)

François Berteloot

Université Paul Sabatier MIG

Institut de Mathématiques de Toulouse

31062 Toulouse Cedex 9

France

berteloo@picard.ups-tlse.fr 